\title{
HOW UNIVERSAL GENERALIZATION WORKS ACCORDING TO NATURAL REASON
}

\author{
KyLe S. Hodge \\ Department of Philosophy. University of South Florida. \\ kshodgedusf.edu
}

\begin{abstract}
Universal Generalization, if it is not the most poorly understood inference rule in natural deduction, then it is the least well explained or justified. The inference rule is, prima facie, quite ambitious: on the basis of a fact established of one thing, I may infer that the fact holds of every thing in the class to which the one belongs-a class which may contain indefinitely many things. How can such an inference be made with any confidence as to its validity or ability to preserve truth from premise to conclusion? My goal in this paper is to explain how Universal Generalization works in a way that makes sense of its ability to preserve truth. In doing so, I shall review common accounts of Universal Generalization and explain why they are inadequate or are explanatorily unsatisfying. Happily, my account makes no ontological or epistemological presumptions and therefore should be compatible with whichever ontological or epistemological schemes the reader prefers.
\end{abstract}

Keywords: universal generalization, reasoning, informal reasoning, informal logic, pedagogy 


\section{Introductory Remarks*}

Universal Generalization is a natural, deductive rule of inference in virtue of which a universal proposition may be validly inferred from a singular proposition which involves a generalized or arbitrary particular. Almost everything turns on what it means for the particular at issue to be "generalized" or "arbitrary." This is because, prima facie, it is a non sequitur to say that because something holds for one case in some class, it must thereby hold for all cases in that class. Common sense seems to dictate that a universal claim may only be validly inferred on the basis of either the conjunction of the proofs conducted on every particular of a class one by one, or else that it follows trivially from some definitions or stipulations which are themselves expressed as universal propositions. And yet, Universal Generalization appears to be a valid inference from a particular to a universal which does neither. How can this be?

The simple fact is that this inference rule is almost universally poorly understood. Even worse, it is almost always poorly and inadequately explained. Although philosophers working today can successfully employ Universal Generalization, their understanding of it seems to remain sufficiently inchoate as to make communication to others ineffectual. A successful and pedagogically useful account of Universal Generalization will be one in which the connection between a singular proposition involving a generalized particular and the corresponding universal proposition is rendered clear and explicit in a finite number of evident parts or steps. Indeed, every canonical rule of natural reasoning ought to be understood in this manner. My intention is to provide an informal remedy to this issue and to make Universal Generalization better and more widely understood by providing a novel and adequate account and justification of it. Moreover, I shall do so in a way which fills the explanatory gaps in extant accounts, and also in a way which does not have epistemological or ontological costs.

\section{Extant Accounts}

It is unfortunate that it is difficult to find a compelling answer to a question that one ought to expect from undergraduate logic students: ${ }^{1}$ how exactly does Universal Generalization work? The extant accounts of Universal Generalization which aim to render the rule intuitive to students of logic are all correct, but they do not do the explanatory work that they should do and which they purport to do. Each account mentioned here is made complete by the solution I shall offer in section 3 .

\footnotetext{
* I want to acknowledge my good friend Jack Short for the stimulating conversation we had which lead to the crucial insights in section 3.3.

1 Cf. Kit Fine's recollection of the genesis of his book-long effort at explaining Universal Generalization in terms of real arbitrary objects (1985b, p. vii).
} 


\section{1- "It Could Have Been Any $x$ " and the Appeal to Arbitrariety}

Perhaps the most common way of explaining why Universal Generalization is valid is because it is evident that the inference from "any $x$ " to "every $x$ " is valid (since they are logically equivalent). If any particular $x$ in class $S$ has property $F$, then every particular in $S$ has $F$. For example, if any marble you pick from the jar will be red, then every marble in the jar is red. It is easy to see why we may infer that every particular in $S$ has $F$ when any (i.e. each) particular in $S$ has $F$. Thus, one reasons as follows:

1. If it could have been any particular $x$ in $S$ which was proven to have $F$, then every particular in $S$ must have $F$. (by logical equivalence of "any" and "every")

2. But it could have been any particular $x$ in $S$ which was proven to have $F$. (intuitive)

3. Therefore every particular in $S$ must have $F$.

This explanation is lacking for want of a way to clearly demonstrate (2). Suppose we try and convince our students that an instance of (2) is true-that any particular triangle may be proven to have internal angles equal to two right angles. We proceed to work the proof on several examples which were seemingly chosen at random. We might even tell the more incredulous of the students: "draw as many triangles as you like, no matter how bizarre they are, and I shall conduct the proof on all of them." ${ }^{2}$ While this dialectical strategy indicates that the unique properties of particular triangles are not important to the proof, this suffices only to communicate the inchoate idea underlying Universal Generalization to another person. This is especially so since this sort of seemingly random selection does not necessarily establish the universality of anything, and may even mislead us to prematurely generalize our results. ${ }^{3}$ Now, the students will likely surmise that they will not be able to find a counterexample to the conclusion that every triangle has internal angles equal to two right angles-but how you deduced that conclusion from a few particular cases remains quite inchoate at best. By failing to explain why the inference from one or many particulars to any or every particular is valid, this approach does little to clarify the procedure of the inference. Thus, an adequate understanding of Universal Generalization cannot be gained by the appeal to "it could have been any $x$."

2 Cf. E. M. Barth (1974, pp. 52-57); you are to imagine yourself simultaneously facing "every possible opponent" armed with a potential counterexample to the proposition as you are conducting the proof (p. 56).

3 For some examples, see: Baez (2018) and Guy (1988). 
A closely related attempt at explanation is the appeal to arbitrariety (also "arbitrary selection" or "selection of an arbitrary particular," etc.). This approach is common in logic textbooks when it comes time to explain Universal Generalization independently of formal rules. ${ }^{4}$ There are usually two ways in which a particular is said to be arbitrary:

1. it was selected at random or with no care as to which particular of the class it is

2. its properties are general and indefinite (whether in fact or in representation)

Each way has its own problems. The issue with (1) is that it is just the warmed over version of the "could have been any" or "could have picked any" approach discussed above. The issue with (2) is that, despite the epistemological and ontological questions it can raise, ${ }^{5}$ it explains Universal Generalization no better than (1) does. Suppose that I have just now concluded Euclid's proof at Elements Book 1, Proposition 32 on an "arbitrary particular triangle" (however you like to conceive of it). Insofar as its properties are general and indefinite, the arbitrary particular triangle either has or represents all and only those properties that every triangle has. As such, the appeal to arbitrariety is simply an appeal to the properties common to all triangles. Yet, trivially, all non-arbitrary particular triangles also have these properties. So the appeal to the arbitrariety of an arbitrary particular triangle is superfluous; as far as the validity of the proof is concerned, whether the particular is "arbitrary" does not matter. Thus, the appeal to arbitrariety does not explain, or even help to explain, the validity of Universal Generalization.

\subsection{Repeatability}

Carlo Cellucci argues for an account of Universal Generalization as being explicable in terms of the repeatability of a proof sequence. ${ }^{6} \mathrm{~A}$ repeatable proof sequence is a proof sequence that produces the same conclusion no matter which particular of the class the proof sequence is applied to. On Cellucci's view, proof sequences are repeatable in virtue of their schematicity: "they are argument-schemata that, given any object in the domain, will yield a proof which is specific to that object." 'As such, Cellucci concludes that "in the universal generalization problem, what is primary is the repeatability of the proof rather than the generalizability of the result. The latter is simply a corollary of repeatability."

\footnotetext{
4 For example, Theodore Sider in Logic for Philosophy (2010, pp. 99, 102) and Irving Copi in Symbolic Logic (1979, p. 72); cf. also the remarks in Cellucci (2009, pp. 4, 8-11).

$5 \mathrm{I}$ am here putting aside the myriad issues with this approach which are sufficient to hinder an easy explanation. See Cellucci (2009, p. 9) for an example.

6 Ibid., pp. 13-15.

7 Ibid., p. 11.

8 Ibid., p. 13.
} 
By inferring universality from repeatability, rather than from generalizability, Cellucci simply relocates the site at which the explanation of Universal Generalization must occur. Originally, the question was how we establish that the conclusion of a given particular proof is generalizable; now, the question is how we establish that a given proof sequence is repeatable with respect to a given class of particular objects. The answer to the latter question is forthcoming: universal propositions form the schematic character of a proof (i.e. they are the argument schemata), and they are derived either from other universal propositions or from definitions. The consequence of this, however, is that there simply is no Universal Generalization. Cellucci promises an explanation of that inference rule, but instead he eliminates it. This is because particulars, which were originally the site of generalization, play no role in the deduction of universal propositions.

As such, we are still left with no explanation of Universal Generalization.

\section{How Universal Generalization Works}

The procedure of universalizing a particular via generalization occurs in three stages:

1. Identification of a particular.

2. Generalization of the particular.

3. Universalization on the basis of the generalized particular.

Let us consider each in turn.

\subsection{Identifying a Particular}

The identification of a particular is the simplest step and usually consists in merely asserting such things as "let $n$ be an integer" or "let $A B C$ be a triangle," or by indicating the same by writing the letter ' $n$ ' or by drawing a triangle whose vertices are labeled 'A,' 'B,' and 'C' respectively. One may also ostend a particular or give a sufficient description of one.

There may be other ways of identifying particulars, e.g. direct experience, modal quantification, etc., but this seems to me to be the most common sort of case; I leave it to the reader to consider others.

\subsection{Generalizing a Particular}

In a particular demonstration that some particular $c$ in some definite class ${ }^{9} S$ has some property $F, c$ is a generalized particular on the condition that every property by virtue of which the particulars in $\mathrm{S}$ could possibly differ is omitted from the premises of the demonstration. ${ }^{10}$

9 A definite class is a class which is defined by a finite set of necessary and sufficient conditions.

10 I was influenced on this matter by George Berkeley's account of Universal Generalization in his A Treatise Concerning 
The particular is said to be generalized inasmuch as every property of $c$ involved in the demonstration is common to all particulars in $S$.

I note that the conclusion of this demonstration on the particular-the singular proposition that $c$ in $S$ has $F$-is itself the premise from which the universal proposition will be validly inferred on the basis of the fact that $c$ is a generalized particular.

\subsection{Universalizing a Generalized Particular: Validly Inferring a Universal Proposition}

Once we have demonstrated of $c$ that it has $F$ in the manner described above, $c$ is such that it cannot differ from any other particular in $S$ with respect to the properties involved in the demonstration because all differentiating properties were omitted. Thus, it follows that it cannot differ from any other particular in $S$ with respect to $F$, for $F$ was itself proven of $c$ on the basis of properties with respect to which the particulars in $S$ cannot differ. So, since $c$ cannot differ with respect to $F$ from any other particular in $S$, it follows that every particular in $S$ must have $F$, for if one thing cannot differ from anything else in the same class with respect to a certain property, it follows that they must all have that property. So we have validly inferred a universal proposition from a singular proposition involving a particular. ${ }^{11}$

This is the rule of inference we call Universal Generalization.

\section{Euclid's Demonstration as Exemplary of Universal Generalization}

It will be instructive to see the procedure that I have laid out above exhibited in an example. For this purpose, we look quite naturally to Euclid. When Euclid constructs his demonstration of Proposition 32 in Book 1 of the Elements, he follows the procedure I have set out.

First, he identifies a particular triangle, $A B C$, by stipulation.

Second, he generalizes $A B C$ by omitting from the premises of the particular demonstration anything concerning the lengths or proportions of the sides or the measures or proportions of the angles. Since these are the only possible ways in which triangles may differ from one another, $A B C$, as it appears in the premises and conclusion of the particular demonstration, is fully generalized.

the Principles of Human Knowledge, Intr. section 16. I extend this Berkeleyan insight by identifying what must be omitted from the premises of a particular demonstration in order to generalize a particular. This addition is vital to clearly articulating, and so explaining, how Universal Generalization works.

11 This may be considered from another point of view. For any universal proposition $\varphi$ which states that all particulars in some class $S$ have some property $F$, a counterexample consists in showing that there is a reason in virtue of which some particular $c$ in $S$ does not have $F$. This reason must be that there is some important difference between $c$ and other particulars in $S$. Since the valid use of Universal Generalization cannot be subject to a counterexample, there must be something in the inference procedure which removes or precludes the sorts of differences that would figure in true counterexamples. So the relevant notion is that $F$ is universal relative to $S$ on the condition that every particular in $S$ cannot differ with respect to $F$. From which comes the procedural method of omitting from the premises of the particular demonstration all properties by virtue of which the particulars in $S$ could differ from one another. Thus, the impossibility of a counterexample coincides with the procedure of generalizing a particular. Hence, the omission procedure grounds and explains the validity of the inference. 
Since Euclid proves of the generalized particular triangle $A B C$ that the sum of its internal angles are equal to the sum of two right angles, he may validly conclude the universal proposition that this fact holds of all triangles. This conclusion is inferred validly because, since all differentiating properties were omitted, $A B C$ must have its properties in common with every triangle. In other words, $A B C$ cannot differ from any other triangle with respect to any property involved in the demonstration. So $A B C$ cannot differ from any other triangle with respect to the sum of its internal angles, for that was the conclusion of the particular demonstration. Thus, because $A B C$ cannot differ from any other triangle with respect to the sum of its internal angles, the sum of the internal angles of every triangle must be equal to two right angles.

\section{Comments on the Foregoing Account}

\subsection{Filling the Explanatory Gaps in Extant Accounts}

My account is able to explain why "it could have been any $x$," as well as why such proof sequences are repeatable.

In a particular demonstration that $c$ in $S$ has $F, c$ may be replaced with $d$ (or any other particular in $S$ ) because the omission procedure is such that any differences between $c$ and $d$ cannot figure in the particular demonstration. So, with respect to the particular demonstration, $c$ and $d$ are the same. Thus, we may rightly say that it could have been $d$, or $e$, or any other particular in $S$.

Furthermore, my account shows why such demonstrations are repeatable. With respect to the particular demonstration, $c$ and any other particular in $S$ are the same. Since each particular in $S$ is the same, the proof conducted on each particular may also be the same. Hence, the same proof sequence may be repeated across numerically distinct particulars because they are the same with respect to the proof.

\subsection{Metaphysical and Epistemological Consequences}

The account I have presented is free of the ontological and epistemological consequences that are typically associated with inferences by Universal Generalization. By means of the omission procedure, one avoids the question of whether there are, or whether we are able to cognize, abstract general ideas (á la Locke) on which we reason to deduce universal conclusions. We likewise avoid the question of whether there "really" exist, in addition to ordinary particulars, such things as arbitrary or indefinite particulars which scholars such as Kit Fine defend. ${ }^{12}$

\footnotetext{
12 Fine says that "in addition to individual objects, there are arbitrary objects: in addition to individual numbers, arbitrary numbers; in addition to individual men, arbitrary men" (1983, p. 55) and that "our talk of arbitrary triangles or indefinite triangles is to be taken at its face value as evincing reference to arbitrary objects" (1985a, p. 57); cf. the exposition in Macnamara (1988, p. 305) and Santambrogio (1988, pp. 630-631).
} 
This account requires only that we deal with ordinary particulars while omitting from our premises the ways in which they can differ from other particulars in the same class; that is all that makes a particular "generalized." It does not require, nor does it preclude, according the particulars any special epistemological or ontological status and it does not require or preclude any extraordinary cognitive powers on our part. Hence, it need not have any epistemological or ontological consequences.

\subsection{Some Important Pedagogical Implications of the Account}

One of the consequences that I am most excited about is the way the account can make this notorious rule of inference clear and explicit to students of logic, mathematics, and critical reasoning. For example, a simple and sound explanation might be:

Let's leave out of the proof every way $A B C$ can differ from other triangles. Then, we can agree that whatever is shown to be true of $A B C$ in the proof must be true of all triangles because things that don't differ must be the same. So, when we do this and show that $A B C$ has internal angles equal to two right angles, we get to infer that every triangle has internal angles equal to two right angles because it is the same as all the other triangles in this respect.

In addition to the inadequate explanations of Universal Generalization discussed in section 2, many students of logic rely, by rote, on the restrictions imposed on the use of the rule in the predicate calculus. My account helps us to see why those restrictions are as they are. The restriction that $\mathrm{Fa}$ must be deduced from premises outside of the particular subproof in which there are no occurrences of $a$ in order for $\forall x F x$ to be validly deduced from $\mathrm{Fa}$ is simply the formal correlate of the omission procedure. As such, an understanding of the inference rule and the formalism which models it can be made to coincide.

\section{Concluding Remarks}

I understand the significance and novelty of this account to consist in the clarity with which it communicates the procedure and validity of Universal Generalization. Extant explanations fail at two specific places. The first is explaining the generalization of a particular or what makes for a generalized particular. The second is explaining the inferential connection between a singular proposition involving a generalized particular and the universal proposition which is inferred on its basis.

Although my account need not have any implications for formal languages employing Universal Generalization, it does have implications for logic or reasoning in the broader sense of thinking or reasoning well. This is because it helps make clear to natural reason 
the manner in which this inference procedure works. When the conditions for successfully applying the inference procedure are clear and evident, it is easier to correctly employ the procedure, detect errors in the attempted use of the procedure by others, and to imaginatively extend it into domains in which others have not recognized its applicability. That is what we should wish for concerning every sort of inference for the sake of extending our stock of knowledge. 
148 - Cogency, Journal of reasoning and argumentation

\section{Bibliography}

Baez, J. (2018). Patterns that Eventually Fail. Azimuth. https://johncarlosbaez.wordpress. com/2018/09/20/patterns-that-eventually-fail/

Barth, E. M. (1974). The Logic of the Articles in Traditional Philosophy: A Contribution to the Study of Conceptual Structures. D. Reidel Publishing Company.

Cellucci, C. (2009). The Universal Generalization Problem. Logique et Analyse, 52(205), 3-20. https://www.jstor.org/stable/44084910

Copi, I. M. (1979). Symbolic Logic (5th ed.). Macmillan Publishing Co., Inc.

Fine, K. (1985a). Natural Deduction and Arbitrary Objects. Journal of Philosophical Logic, 14(1), 57-107. https://doi.org/10.1007/BF00542649

Fine, K. (1985b). Reasoning With Arbitrary Objects. Basil Blackwell.

Fine, K., \& Tennant, N. (1983). A Defense of Arbitrary Objects. Proceedings of the Aristotelian Society, Supplementary Volumes, 57(1), 55-90. https://doi.org/10.1093/ aristoteliansupp/57.1.55

Guy, R. K. (1988). The Strong Law of Small Numbers. The American Mathematical Monthly, 95(8), 697-712. https://doi.org/10.2307/2322249

Macnamara, J. (1988). [Review of the book Reasoning With Arbitrary Objects, by K. Fine]. The Journal of Symbolic Logic, 53(1), 305-306. https://doi.org/10.2307/2274449

Santambrogio, M. (1988). [Review of the book Reasoning With Arbitrary Objects, by K. Fine]. Noûs, 22(4), 630-635. https://doi.org/10.2307/2215463

Sider, T. (2010). Logic for Philosophy. Oxford University Press. 